\title{
The Influence of Double Acting Hydraulic Piston on Tractive Forces for Towing Vehicle on Steering of Wagon Type Articulated Tracked Vehicle
}

\author{
Ekhlas A. Basher \\ College of Electronics Engineering / Electronic Engineering Department
}

\begin{abstract}
Tracked vehicles have been in use for many years ago thus, there has been increasing interest in articulated tracked vehicles for various applications such as they can be used as transporters for heavy equipment or as heavy earth-moving machines.

Generally, it has been accepted that the wagon-type articulation of tracked vehicles is the simplest type of articulation. However, previous analysis has shown that this kind of articulation creates a problem to steering in the straightening-up position. This paper deals theoretically with the possibility of changing the hydraulic piston, which used now in articulated tracked vehicles by a double acting hydraulic piston as another solution. It explains the effect of two forces of the piston on the outside tractive force and inside tractive force for the towing vehicle, when using a conventional wagon-type articulation of tracked vehicles with the introduction of double acting hydraulic piston in the system. It show that the length to the width ratio of the towing tracked vehicle is the main factor that affects much more than the other on the forces of the towing vehicle, where the value of this ratio (less than one) gives a good straighting up.

Keywords: tracked vehicles, articulation of tracked vehicles, thrust force, hydraulic piston

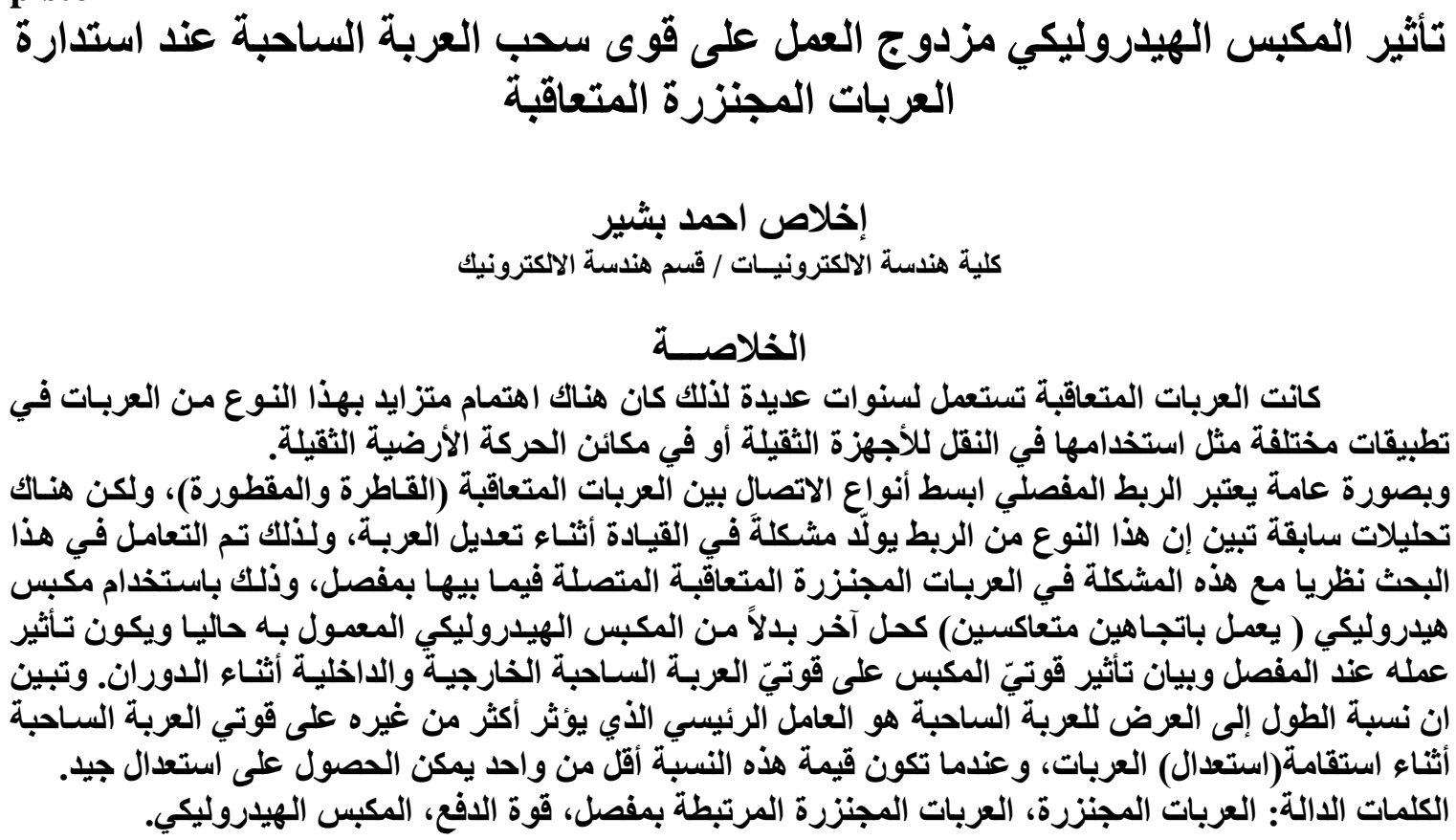

Received: 14 - 6 - 2010

Accepted: $15-11-2011$ 


\section{1- Notation:}

$h$ Width of the towing tracked vehicle. $\theta$

$h_{1}$ Width of the towed tracked vehicle.

$d$ Distance between the hinge point and centre of the gravity of the towed vehicle.

$F_{\text {out }}$ Outside track force for the towing vehicle.

$F_{\text {in }}$ Inside track force for the towing vehicle.

$F_{p}$ Piston force.

$F_{h}^{p}$ Lateral hinge force with using the piston.

$V \quad$ Lateral hinge force without using the piston.

$l \quad$ Track length of the towing vehicle.

$l_{1} \quad$ Track length of the towed vehicle.

$x$ Distance of the hinge point to the centre ofgravity of the towing vehicle.

$y$ Offset of the hinge point from the centreline towards the inside track.

$W$ Towing vehicle weight.

$W_{1}$ Towed vehicle weight.

$r$ Radius of turn of the vehicle. $\theta \quad$ Angle between the centre lines of the two vehicles.

$\mu \quad$ Coefficient of friction between ground and vehicle tracks.

$\mu_{r} \quad$ Coefficient of friction of rolling resistance.

$K \quad$ Dimensionless factor representing $V / \mu W$.

$R_{l h} \quad$ Dimensionless factor representing $l / h$.

$R_{d l_{1}}$ Dimensionless factor representing $d / l_{1}$.

$R_{x h} \quad$ Dimensionless factor representing $x / h$.

$R_{y h} \quad$ Dimensionless factor representing $y / h$.

$\psi_{v} \quad$ Dimensionless factor representing $V / \mu W_{1}$.

$\psi_{\text {out }}$ Dimensionless factor representing $F_{\text {out }} / \mu W$.

$\psi_{\text {in }} \quad$ Dimensionless factor representing $F_{\text {in }} / \mu W$.

$\psi_{p} \quad$ Dimensionless factor representing $F_{p} / \mu W$.

\section{2- Introduction:}

Tracked vehicles have been used for years ago, and used in many applications such as military, construction, and agricultural, Bekker [1]. Many studies dealing with tracked vehicles have been done and published since then; nevertheless, there are only a few of them relevant to our interest. Bekker $[1,2,3]$.

Kitano and Jyozaki [4] investigated the problems concerning steerability, stability, and relevant behavior of a tracked vehicle operated at high speeds on level paved ground. They developed equations of uniform turning motions of the tracked vehicle based on both the assumption that the pressure distribution beneath both tracks are modeled as concentrated forces acting under each road wheel, and the assumption that the friction between ground and track is Coulomb friction and constant. Subsequently, Kitano and Kuma [5] presented a mathematical model of the non-stationary motion of a tracked vehicle maneuvered on level hard ground. The model employed a pull-slip equation obtained from pull-slip tests to approximate the friction coefficient varying with the amount of track slip, and was simulated using a digital computer for the analysis and prediction of steering characteristics of tracked vehicles under different operating conditions.

Alhimdani [6] dealt theoretically with the wagon-type articulation of tracked vehicles. It is a particular kind of articulation in which a tracked vehicle tows a tracked trailer by a drawbar which is rigidly fixed to the trailer, and is hinged on the towing vehicle. Alhimdani and Younis [7] dealt theoretically with the wagon-type articulation but with the introduction of hydraulic piston to see the effect of the piston force on the hinge force. Alhimdani [8] is employed the mathematical models of steering articulated tracked vehicles and terrain inputs are available, they could fit into the methodological framework presented here so that a more comprehensive picture. 
Kitano's tracked vehicle model, including two other less sophisticated models, slightly modified and evaluated by Ehlert, et al. [9]. The simulation results from the modified models were verified by comparison with field measurement data and among the simulation results from different models themselves. They summarized that the Kitano model could be used to closely predict the radius of turning of tracked vehicles. Furthermore, radius enlargement factors could be determined by means of the Kitano model, and be utilized to improve accuracy of the other two models.

Murakami, et al. [10] proposed a mathematical model, which predicts the spatial motion of a tracked vehicle on non-level soft terrains, and an advanced soil-track interaction model, based upon soil plasticity theories.

Shiller and Serate [11] presented a dynamic model of a tracked vehicle by assuming Coulomb friction between tracks and ground, uniform normal pressure distribution under the tracks, and the center of mass located at the geometric center of the vehicle. The model was employed in their work for computing the track forces and track speeds of the tracked vehicle, which are required to follow a given path at specified speeds on horizontal and inclined planes. Recently, Le, et al. [12] developed a relatively simple model of tracked vehicle based upon Shiller's model. The model was improved by adopting a more accurate soil model and taking into account all resistive ground forces.

Martínez, J.L., et. al, [13] proposed a kinematic approach for tracked vehicles in order to improve motion control and pose estimation. Complex dynamics due to slippage and soil shearing make it difficult to predict the exact motion of the vehicle from the velocity of the two tracks. Martínez, J.L., et. al, [14] the tractor to avoid interunit collision during forward motion. This method can be used for systems that combine any type of on-axle and off-axle joints. We propose an algorithm to compute curvature bounds based on the analysis of steady and transient responses. These limitations can be introduced at the path tracking and path planning levels for autonomous navigation. Thus, the tractor can be controlled to follow admissible paths much in the same way as when it does not tow any trailer. Martínez, J.L., et. al, [15] extended previous work of backward motion for off-axle trailers. To this end, the last trailer is considered to behave as a virtual tractor. Then, virtual steering is computed by applying any path tracking method for single nonholonomic mobile robots, incorporating limitations if necessary. Finally, kinematic relationships are used to translate motion commands to the actual tractor. The method has been validated experimentally with two heterogeneous off-axle trailers attached to the tracked mobile robot Auriga- $\alpha$.

Fijalkowski [16] considers a novel tri-mode hybrid mobility and tri-mode hybrid steerability concept of A-E IATVs with Drive-By-Wire (DBW) All- Wheel-Driven (AWD) tracks. The IATV's unique Electro-Mechanic (E-W steering mechanism involves articulatian of the front and rear IATV's hull tracked-units in relation to each other. In order to clarify the turning features, the physical model and mathematical model may be presented. The IATV's power plant is arranged in the first IATV's hull tracked-unit behind the Human Driver's (HD) cabin. From the Gas Turbine-Generator, Motor (GT$\mathrm{G} / \mathrm{M}$ ) that based on the Fijalkowski Turbine Boosting (FTB) system, or the 2- 4- or even 5-stroke, thermo-dynamic cycle, twin-opposed-piston, crankless Internal Combustion Engine (ICE), called the Fijalkowski Engine-Generator/Motor (FE-G/M). The output electric energy is supplied to DBW AWD tracks ' EM wheel-hub motors af all the sprocket-, road- and tensioner- wheels of the front and rear IATV's hull tracked units. 


\section{3- The Theoretical consideration:}

A study of mechanics of tracklayer steering would include development of a theory which would relate the tractive thrust from each track to the radius of turn (steering angle). It was considered in this work a start with the simplest situation of articulated steering. A conventional track-laying vehicle towing an undriven tracked trailer using wagon-type articulation. Effects of gradient, rolling, inertia and centrifugal forces have been neglected. In addition, a uniform pressure distribution and constant coefficient of lateral friction have been assumed.

\subsection{Towed tracked trailer analysis:}

A tracked trailer being towed by a rigid drawbar is shown in Fig. 1. The drawbar is hinged on the back of a powered tracked vehicle. Towed vehicle represents the tracked trailer, or any other tracked vehicle with its engine out of power, like a knocked-out tank.

Frictional force/ unit length $=\frac{\mu W_{1}}{l_{1}}$

(combining both tracks)

Total frictional force acting outwards $=\frac{\mu W_{1}}{l_{1}} m$

Total frictional force acting inwards $=\frac{\mu W_{1}}{l_{1}}\left(l_{1}-m\right)$

$\sum F_{y}=0$

$V=\frac{\mu W_{1}}{l_{1}}\left(2 m-l_{1}\right)$

$\sum M=0$

Taking moments about the hinge point, $\frac{\mu W_{1}}{l_{1}}\left(l_{1}-m\right)\left(d+\left(m-\frac{l_{1}}{2}\right)+\frac{\left(l_{1}-m\right)}{2}\right)-\frac{\mu W_{1}}{l_{1}}(m)\left(d-\frac{l_{1}}{2}+\frac{m}{2}\right)=0$

Therefore,

$m^{2}+m\left(2 d-l_{1}\right)-d l_{1}=0$

and,

$m=\frac{-2 d+l_{1} \pm \sqrt{\left(4 d^{2}+l_{1}^{2}\right)}}{2}$

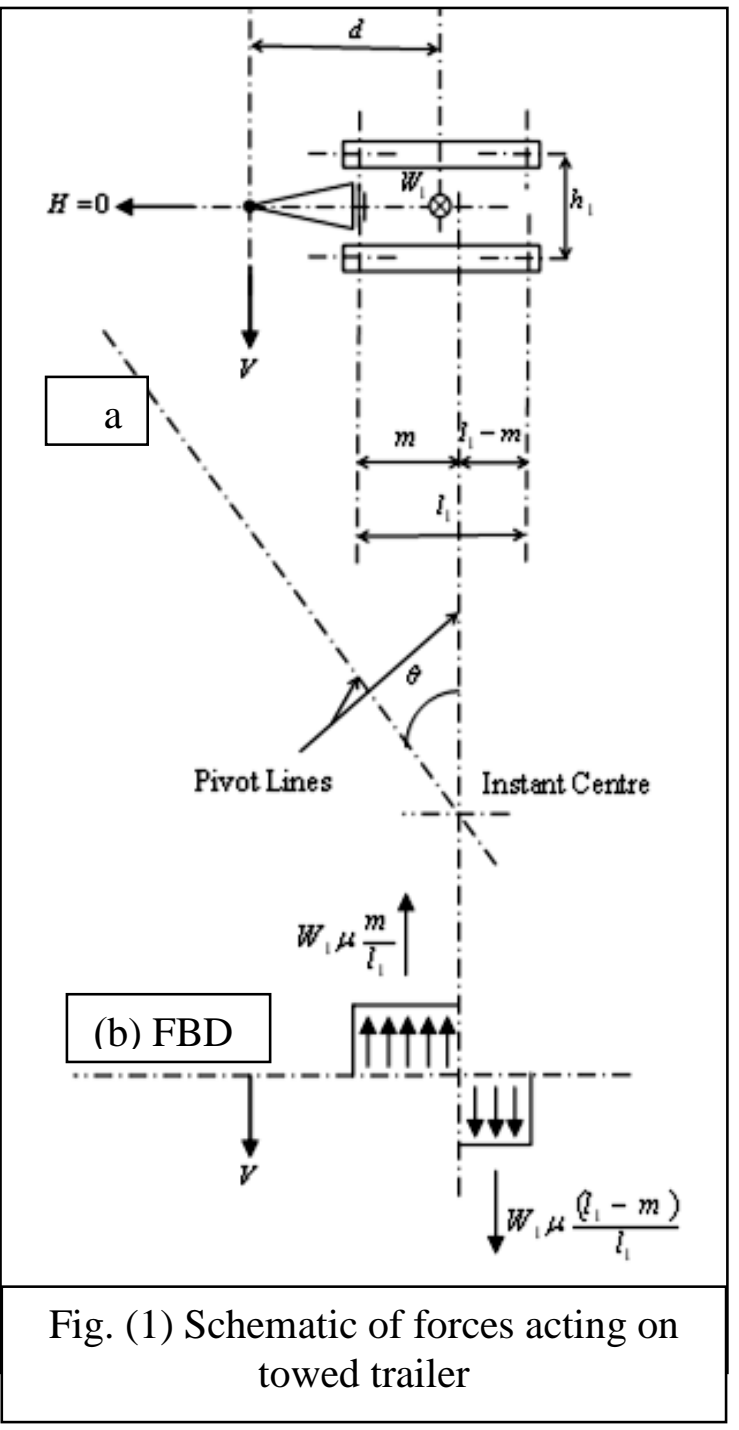

Taking the positive values of $m$ and introducing the dimensionless quantities $R_{d l_{1}}$ and $\psi_{v}$, then,

$\psi_{v}=\frac{V}{\mu W_{1}}=-2 R_{d l}+\sqrt{\left(2 R_{d l}\right)^{2}+1}$

The values of $\psi_{v}$ show how the hinge force $V$ varies with maximum attainable value of $V$ which is equal to $\mu W_{1}$. 


\subsection{Towing tracked vehicle analysis:}

The braking and tractive forces $F_{\text {in }}$ and $F_{\text {out }}$ must be taken into account when dealing with the front vehicle analysis. The vehicle is powered tracked unit using its engine to steer the twovehicle system. The forces involved are shown in (Fig. 2).

$\sum F_{y}=0$

$V \cos \theta+\frac{\mu W}{l}(l-n)=\frac{\mu W}{l} n$

$\sum F_{x}=0$

$F_{\text {out }}+F_{\text {in }}-V \sin \theta=0$

$\sum M=0$;

$\left(F_{\text {out }}-F_{\text {in }}\right) \frac{h}{2}+V \cos \theta\left(x+\frac{l}{2}-\frac{l-n}{2}\right)$

$-\frac{\mu W n}{l}\left(\frac{n}{2}+\frac{l-n}{2}\right)=0$

Eliminating $n$ and introducing the dimensionless quantities

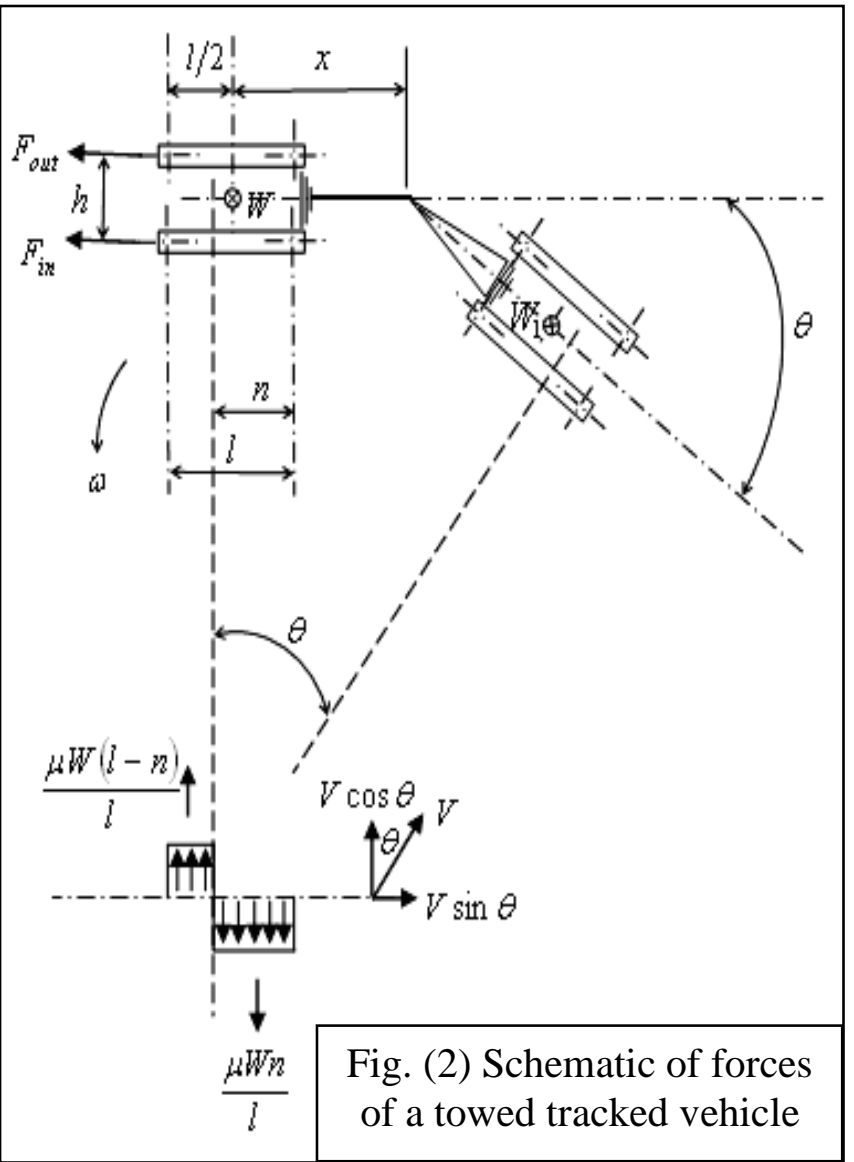
$\psi_{\text {out }}, \psi_{\text {in }}, K, R_{x h}$, and $R_{l h}$ then,

$\psi_{\text {out }}=\frac{F_{\text {out }}}{\mu W}=K\left(0.5 \sin \theta-R_{x h} \cos \theta\right)+0.25 R_{\text {lh }}\left(1-K^{2} \cos ^{2} \theta\right)$

$\psi_{\text {in }}=\frac{F_{i n}}{\mu W}=K\left(0.5 \sin \theta+R_{x h} \cos \theta\right)-0.25 R_{l h}\left(1-K^{2} \cos ^{2} \theta\right)$

Where: $R_{x h}=\frac{x}{h}, R_{l h}=\frac{l}{h} \& K=\frac{V}{\mu W}$

The value of $K$ represents the variation of the towed vehicle weight with the towing vehicle weight, with respect to $\psi_{v}$.

However, this analysis is concerned with vehicle changes from moving along a circle of a certain radius onto a circle of a smaller radius .It will continue on this smaller path until the new direction is approached and it will then have to straighten up. The mechanics of the straightening up becomes different from that of the initial turning.

The main difference in the two situations is that force through the hitch point of the front vehicles is in the opposite direction to the normal turning case as shown in fig. (3).

This means that the turning line will be shifted backwards behind the centre of gravity instead of being in front of it. The towed tracked trailer will have exactly the same analysis as before. The front tracked vehicle will have a slightly different analysis leading to the following equations: 


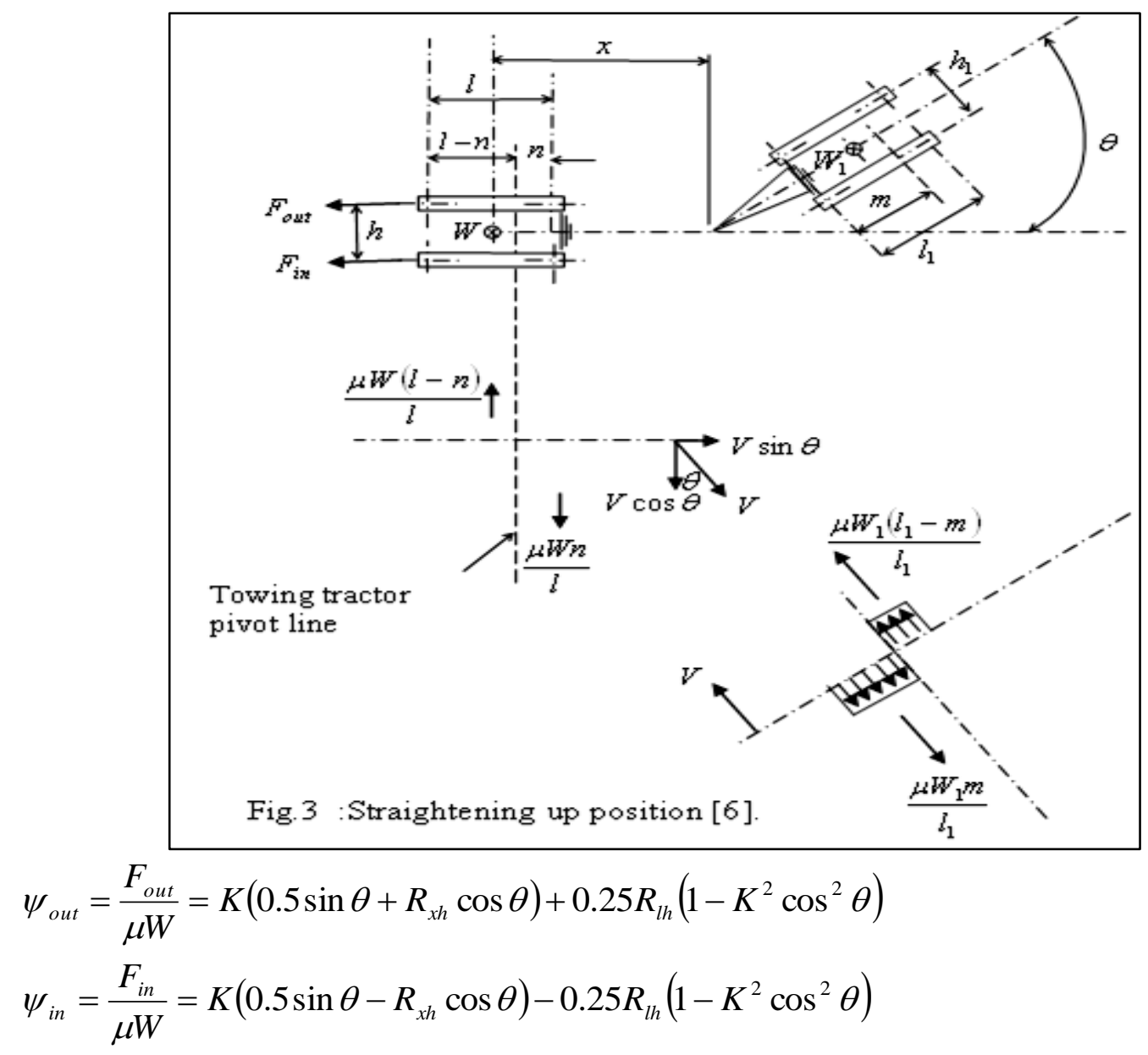

The above tow equations can be used for any case of the straightening-up position.

\section{4- The Double Acting Hydraulic Piston:}

Pumps perform the function of adding energy to the fluid of a hydraulic system for transmission to some output location. Hydraulic cylinders extract energy from the fluid and convert it to mechanical energy to perform useful work.It usually consists of a moveable element such as a piston and piston rod within a cylinder bore. A fluid power cylinder is a linear actuator, which is most useful and effective in converting fluid energy to an output force in a linear direction for performing work such as pulling or pushing in a variety of engineering applications such as in machine tools and other industrial uses [17].

\section{Construction and Operation of Cylinder:}

A double acting hydraulic cylinder consists of the following major parts as shown in Fig. (4).

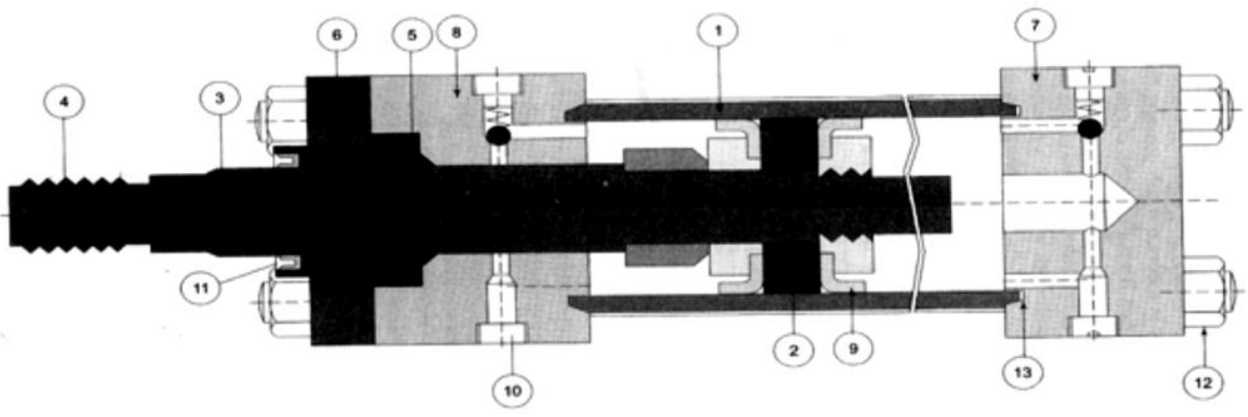


Fig. 4: The Typical Construction details of Double acting hydraulic cylinder. [17]
1. Cylinder body or tube
2. Piston
3. Piston rod
4. Threaded end piston rod.
5. Bush seal
6. Bush

7. Front end cover or head end

8. Rear end covers or cap end or rod end. 9. Piston seal

10. Cushioning assembly

12. Tie-rod fastener

11. Rod wiper

13. Circular groove for wire to join tube and end cover.

The figure shows the constructional details of a double acting hydraulic cylinder. Such a cylinder can be extended and retracted hydraulically. Thus, an output force can be applied in two directions (extension and retraction). This particular cylinder has a working pressure of $14 \mathrm{~kg} / \mathrm{cm}^{2}$. In this design, the barrel is made of seamless steel tubing, honed to a fine finish on the inside. The piston, which is made of ductile iron, contains U-cup packings to seal against leakage between the piston and barrel. The ports are located in the end caps, which are secured to the barrel by tie rods. The tapered cushion plungers provide smooth deceleration at both ends of the stroke. Therefore, the piston does not bang into the end caps with excessive impact, which could damage the hydraulic cylinder after a given number of cycles [17].

A double-acting cylinder can be extended and retracted hydraulically. Thus, an output force can be applied in two directions (extension and retraction). This type of cylinder has working pressure rating of 40 bar to 400 bar depending on the constructional details and application needs. The output force is proportional to the bore area.

Double-acting cylinders sometimes contain cylinder cushions at the ends of the cylinder to slow the piston down near the ends of the stroke. This prevents excessive impact when the piston is stopped by the end caps. Deceleration starts when the tapered plunger enters the opening in the cap. This restricts the exhaust flow from the barrel to the port. During the last small portion of the stroke, the oil must exhaust through an adjustable opening. The cushion design also incorporates a check valve to allow free flow to the piston during direction reversal [18].

\section{5- Effect of Double Acting Hydraulic Piston:}

In this work, the possibility of changing the hydraulic piston which is used now in articulated tracked vehicles by a double acting hydraulic piston was studied, in order to improve the steering and the straightening-up these vehicles by finding the effect of the double acting hydraulic piston on the tractive forces of towing vehicle.

The development of a theory relating the tractive thrust from each track and wagon hinge force with the radius of turn (or steering angle) have been achieved in section (3). Effects of gradient, rolling resistance, inertia and centrifugal forces have been neglected. In addition a uniform pressure distribution and constant coefficient of lateral friction have been assumed.

The object of this analysis is to compare the required hinge force when using a conventional wagon-type articulation of tracked vehicles with the hinge force needed when introducing 2- way hydraulic piston in the system as shown in fig. (5).

A tracked trailer being towed by a rigid draw bar is shown in (Fig.2). The draw bar is hinged on the back of a powered tracked vehicle. The towed vehicle represents the tracked trailer or any other tracked vehicle with its engine out of action. 


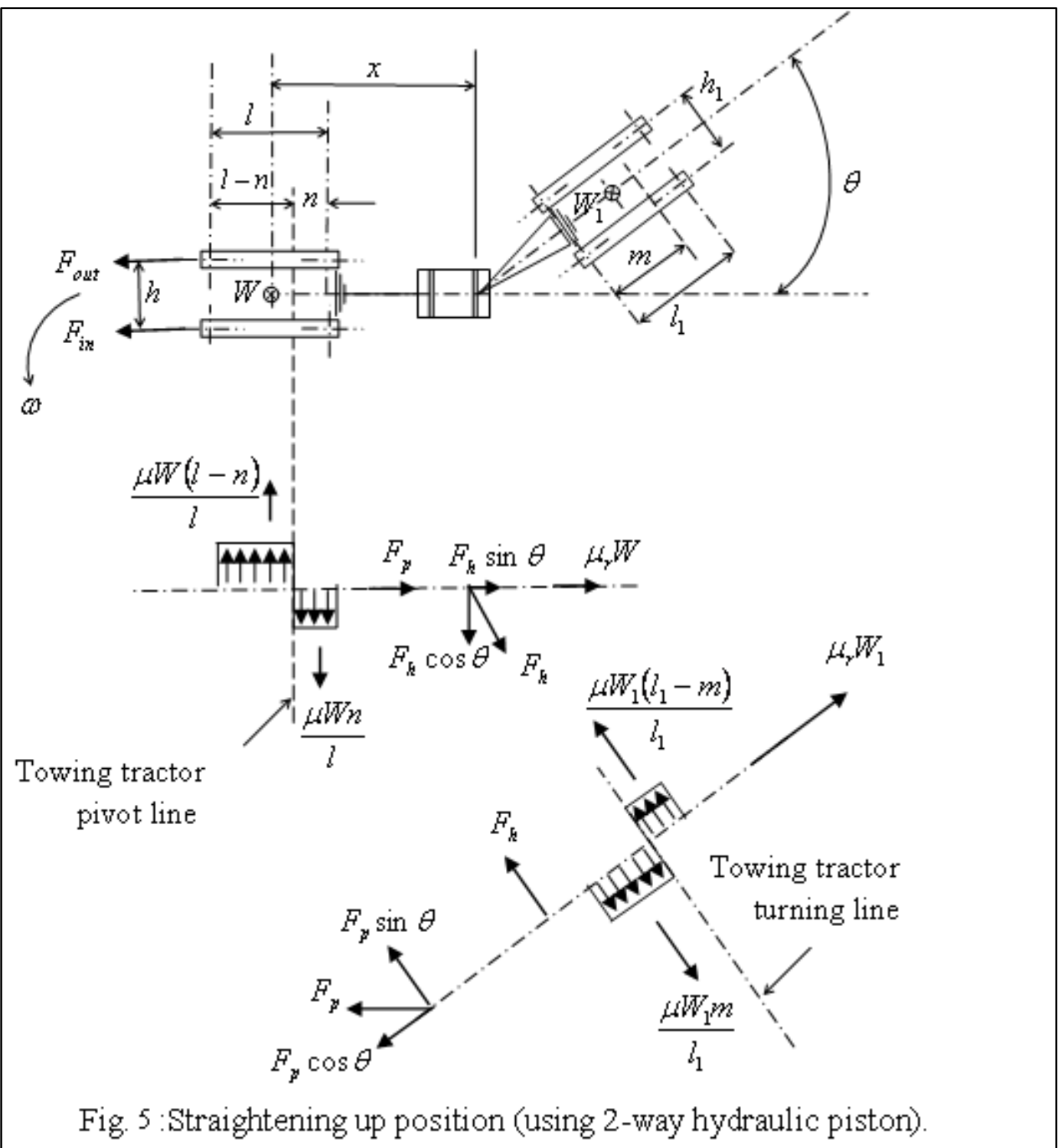

\subsection{The Kinetic Equations:}

For towed vehicle:

$\sum F_{y}=0$

$F_{h}+F_{p} \sin \theta+\frac{\mu W_{1}}{l_{1}}\left(l_{1}-m\right)-\frac{\mu W_{1}}{l_{1}} m=0$

$\sum F_{x}=0$

$F_{p} \cos \theta=\mu_{r} W_{1}$

or $\quad F_{p}=\frac{\mu_{r} W_{1}}{\cos \theta}$

Taking moments about the hinge point, $\quad \sum M=0$

$\frac{\mu W_{1}}{l_{1}}\left(l_{1}-m\right)\left(d+\left(m-\frac{l_{1}}{2}\right)+\frac{l_{1}-m}{2}\right)-\frac{\mu W_{1}}{l_{1}} m\left(d-\frac{l_{1}}{2}+\frac{m}{2}\right)=0$ 
or $\quad m=\frac{-2 d+l_{1} \mp \sqrt{4 d^{2}+l_{1}^{2}}}{2}$

For towing vehicle:

$$
\begin{aligned}
& \sum F_{x}=0 \\
& F_{\text {out }}+F_{\text {in }}-F_{p}-F_{h} \sin \theta-\mu_{r} W=0 \\
& \sum F_{y}=0 \\
& \frac{\mu W(l-n)}{l}-\frac{\mu W n}{l}-F_{h} \cos \theta=0
\end{aligned}
$$

From the equations $(5,6,7,8 \& 9)$, we get:

$$
n=\frac{l}{2 W}\left(W-W_{1} Z \cos \theta+\frac{\mu_{r}}{\mu} W_{1} \sin \theta\right)
$$

Where

$$
Z=-2 R_{d l_{1}} \mp \sqrt{4 R_{d l_{1}}^{2}+1}
$$

$\sum M=0$

$\left(F_{\text {in }}-F_{\text {out }}\right) \frac{h}{2}+\frac{\mu W}{l}(l-n)\left(x+\frac{l}{2}-\frac{l-n}{2}\right)-\frac{\mu W n}{l}\left(x-\frac{l}{2}+\frac{n}{2}\right)=0$

By solution above equations, we get:

$$
\begin{array}{r}
\psi_{\text {out }}=\frac{\mu_{r}}{\mu} \frac{W_{1}}{W}\left(0.5 \cos \theta-\left(R_{x h}-0.5 R_{l h}\right) \sin \theta\right)+\frac{W_{1}}{W} Z\left(0.5 \sin \theta+\left(R_{x h}-0.5 R_{l h}\right) \cos \theta\right) \\
+0.5 \frac{\mu_{r}}{\mu}+0.5 R_{l h}-\frac{R_{l h}}{4 W^{2}}\left(W-W_{1} Z \cos \theta+\frac{\mu_{r}}{\mu} W_{1} \sin \theta\right)^{2}
\end{array}
$$

and

$$
\begin{array}{r}
\psi_{\text {in }}=\frac{\mu_{r}}{\mu} \frac{W_{1}}{W}\left(0.5 \cos \theta+\left(R_{x h}-0.5 R_{l h}\right) \sin \theta\right)+\frac{W_{1}}{W} Z\left(0.5 \sin \theta-\left(R_{x h}-0.5 R_{l h}\right) \cos \theta\right) \\
+0.5 \frac{\mu_{r}}{\mu}-0.5 R_{l h}+\frac{R_{l h}}{4 W^{2}}\left(W-W_{1} Z \cos \theta+\frac{\mu_{r}}{\mu} W_{1} \sin \theta\right)^{2}
\end{array}
$$

and

$\psi_{p}=\frac{F_{p}}{\mu W}=\frac{\mu_{r}}{\mu} \frac{W_{1}}{W} \frac{1}{\cos \theta}$

\section{6- The Results and Discussion:}

Computer is found necessary for solving the equations to find the results. Several variables are found in equations (11, 12 and 13). These variables are ( $\left.R_{d l_{1}}, \mu_{r} / \mu, R_{x h}, R_{l h} \& W_{1} / W\right)$, accordingly , to examine the individual effect of each variable four of them are kept fixed while the fifth is made variable, so that the values which represents the performance of the system are calculated, namely $\psi_{\text {out }}, \psi_{\text {in }} \& \psi_{p}$. 
Fig. (6), [6], shows the general trend for steering the conventional system, which is an ideal system in comparison to a particular wagon-type articulated system, (towing system). It would be desirable if the ideal system can be achieved. As, the track thrusts are fairly close to the zero thrust line, thus the tractive effort and the thrust on the terrain are both minimal.

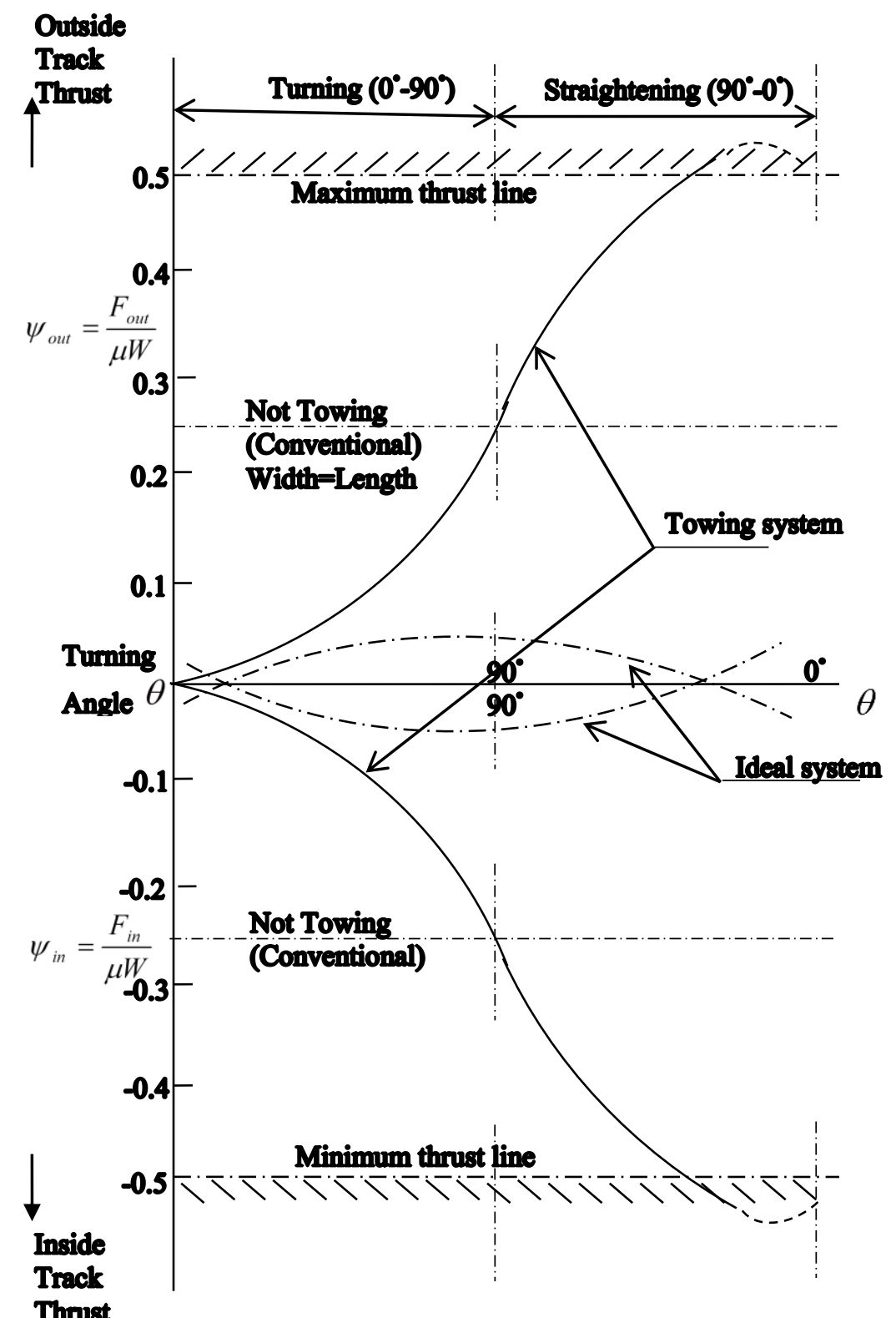

Fig. 6: Comparison of thrusts for different systems [6].

In addition, the theoretical values, obtained by the above-mentioned reference, have been evaluated for different values of steering angle. The dimensionless values of the thrust forces expressed by $\left(\psi_{\text {out }}\right.$ and $\left.\psi_{\text {in }}\right)$ are to be located in the range between $(0.5 \&-0.5)$ giving the maximum and minimum thrust line in the design. The previous range of thrust lines are also adopted in references [7,8]. It is to be mentioned that, in the present work, not only $\psi_{p}$ are determined but rather other results reflecting the tractive force $\left(\psi_{\text {out }}\right.$ and $\left.\psi_{\text {in }}\right)$ are also necessary evaluated for different parameters, as explained earlier, where the maximum and minimum thrust lines of, fig. (6), are taken as reference to obtain the best results. Clearly, $\psi_{p}$ is directly proportional to $\left(\mu_{r} / \mu \& W_{1} / W\right)$ while the effect of angle $\theta$ as governed by the term $(1 / \cos \theta)$, however the effect of an increase in angle $\theta$ would give an increase in $\psi_{p}$. 
In this work, the factor $R_{l h}$ is taken equal to unity in cases (1 to 14), while it is taken less than unity in cases $(15,16)$ as shown in figures $(21,22)$.

Four values of the variable $\mu_{r} / \mu$ have been taken as different cases. There values are $(0.1,0.2,0.3 \& 0.4)$ while the dimensionless factors $\left(R_{d l}, R_{x h}, R_{l h} \& W_{1} / W\right)$ are mentioned as constants in the calculation. In order to examine the effect of the variable $\mu_{r} / \mu$ on the system tractive forces a number of plots are obtained from the calculation, figures $(7,8,9 \& 10)$. It may be noticed that, for $\mu_{r} / \mu=0.1$, minimum values of these forces are obtained, which suggests that decreasing the factor $\mu_{r} / \mu$ should improve the values of the tractive forces.

Further, another four different cases in which different values of the parameter $R_{d l}$ are considered as $(0.3,0.5,0.6 \& 1.5)$ respectively are used in the calculation. Keeping the values of $\left(\mu_{r} / \mu=0.1\right)$ and $\left(R_{x h}, W_{1} / W\right)$ as constants, the effect of the factor $R_{d l_{1}}$ is investigated. The results shows that by increasing the value of $R_{d l}$ gives an improvement on the tractive forces, as shown in figures $(1112,13 \& 14)$. To examine the effect of factor $R_{x h}$, three different values are used in the calculation, these values are (1.5, $0.6 \& 0.3)$ respectively, Here, the other values are taken as $\left(\mu_{r} / \mu=0.1\right)$, and $\left(R_{d l_{1}}=1.5\right)$, the results of which are summarized in figures $(15,16 \& 17)$, which indicates that, by decreasing the value of $R_{x h}$ the calculated values of the system tractive force are improved and best results are obtained for $R_{x h}=0.3$.

Now, by fixing the best obtained values of the three different design parameters as ( $\mu_{r} / \mu=0.1, R_{d l}=1.5$ and $\left.R_{x h}=0.3\right)$ and taking three different cases of the dimensionless factor $W_{1} / W$ as $(0.75,0.5 \& 0.3)$, the values of the tractive forces are improved for smaller value of $W_{1} / W$; these are clear in figures $(18,19 \& 20)$.

It may be noticed that by taking $R_{l h}$ less than unity for example, $(0.5,0.75)$ better values of the tractive forces in the towing vehicle are obtained for smaller values of $R_{l h}$, as shown in the figures $(21,22)$ respectively.

This is not recommended, since, in practice, $R_{l h}$ cannot be taken less than unity as the vehicle width is always smaller than the length.

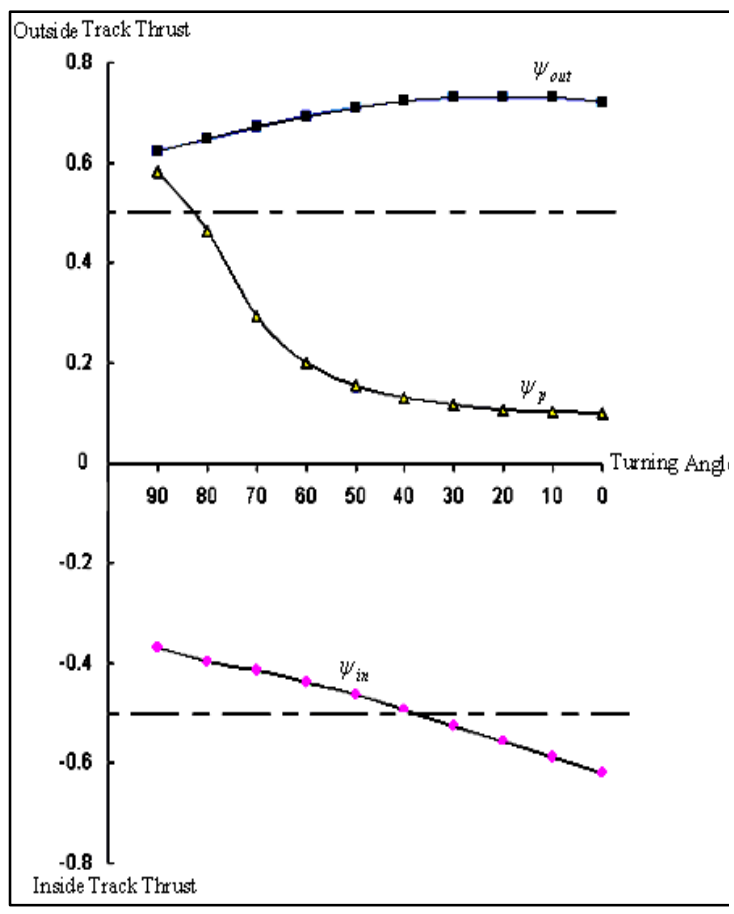

Fig. (7):

Case $1\left(\frac{\mu_{r}}{\mu}=0.1, R_{d l_{1}}=1, R_{x h}=1 \& \frac{W_{1}}{W}=1\right)$

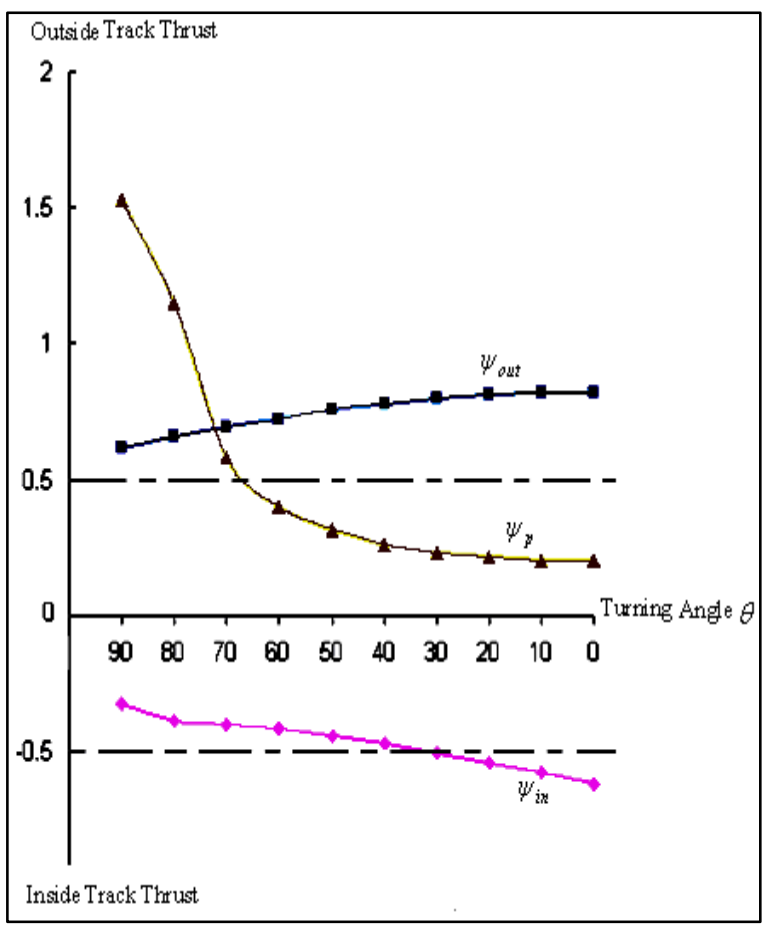

Fig. (8):

Case $2\left(\frac{\mu_{r}}{\mu}=0.2, R_{d l_{1}}=1, R_{x h}=1, \frac{W_{1}}{W}=1\right)$ 


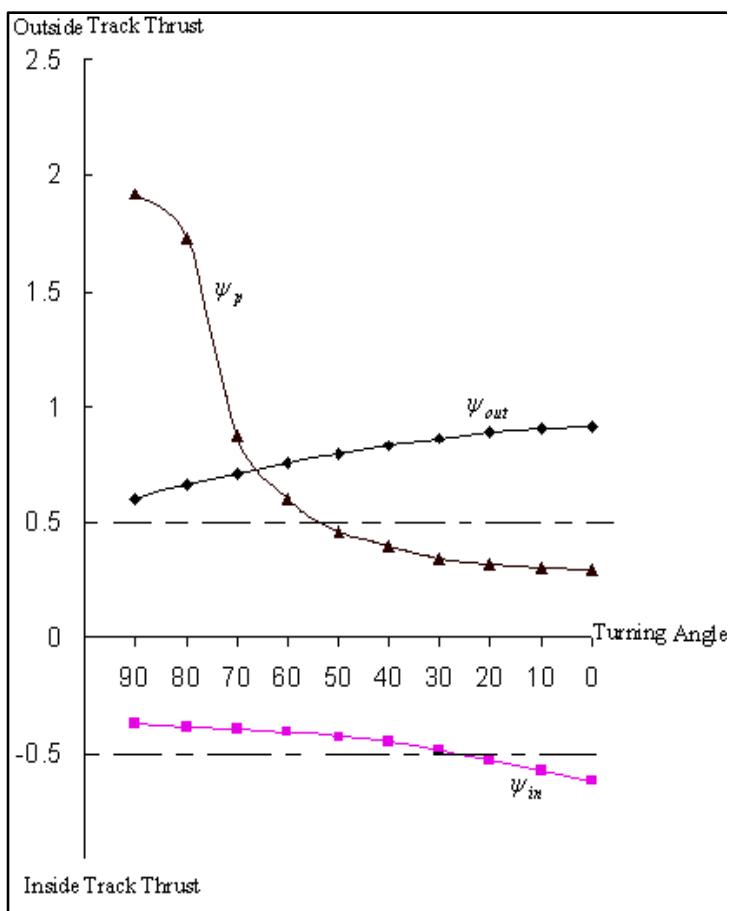

Fig. (9):

Case $3\left(\frac{\mu_{r}}{\mu}=0.3, R_{d l_{1}}=1, R_{x h}=1, \frac{W_{1}}{W}=1\right)$

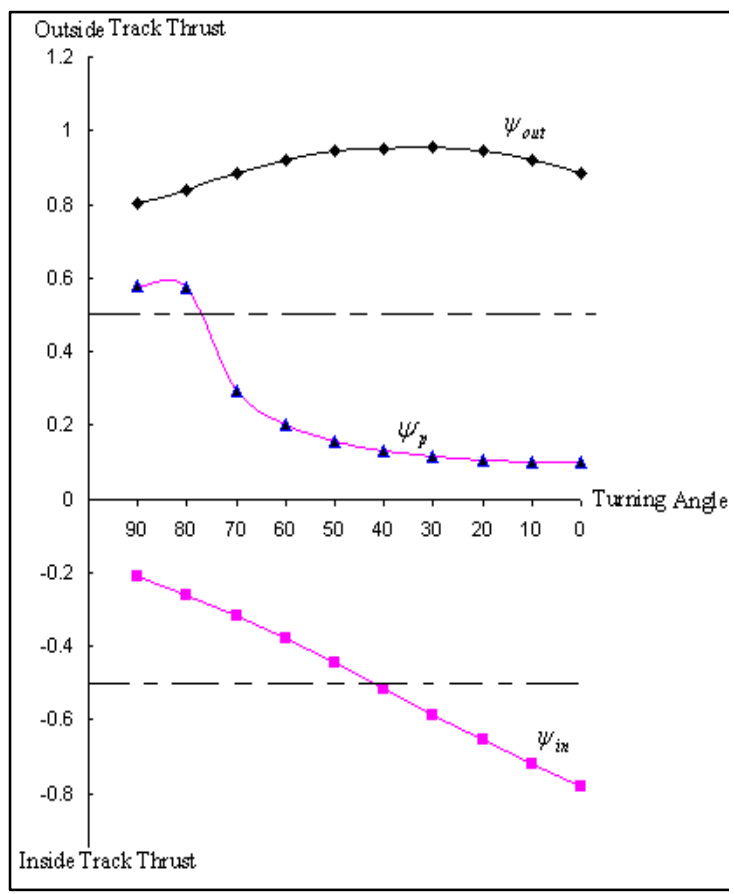

Fig. (11):

Case $5\left(\frac{\mu_{r}}{\mu}=0.1, R_{d l_{1}}=0.3, R_{x h}=1 \& \frac{W_{1}}{W}=1\right)$

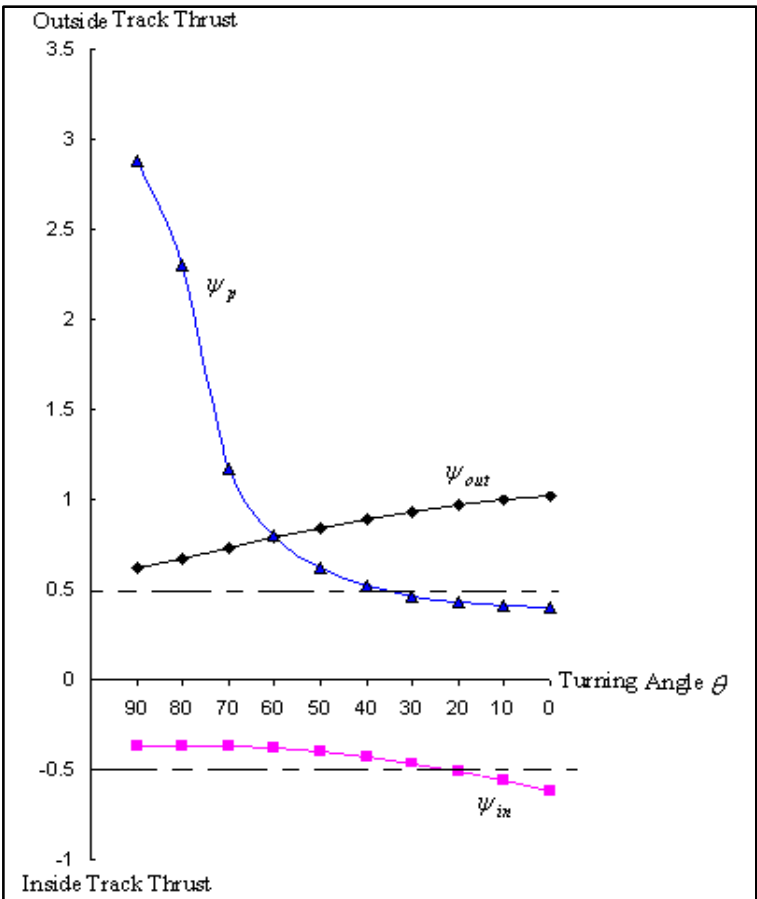

Fig. (10)

Case $4\left(\frac{\mu_{r}}{\mu}=0.4, R_{d l_{1}}=1, R_{x h}=1, \frac{W_{1}}{W}=1\right)$

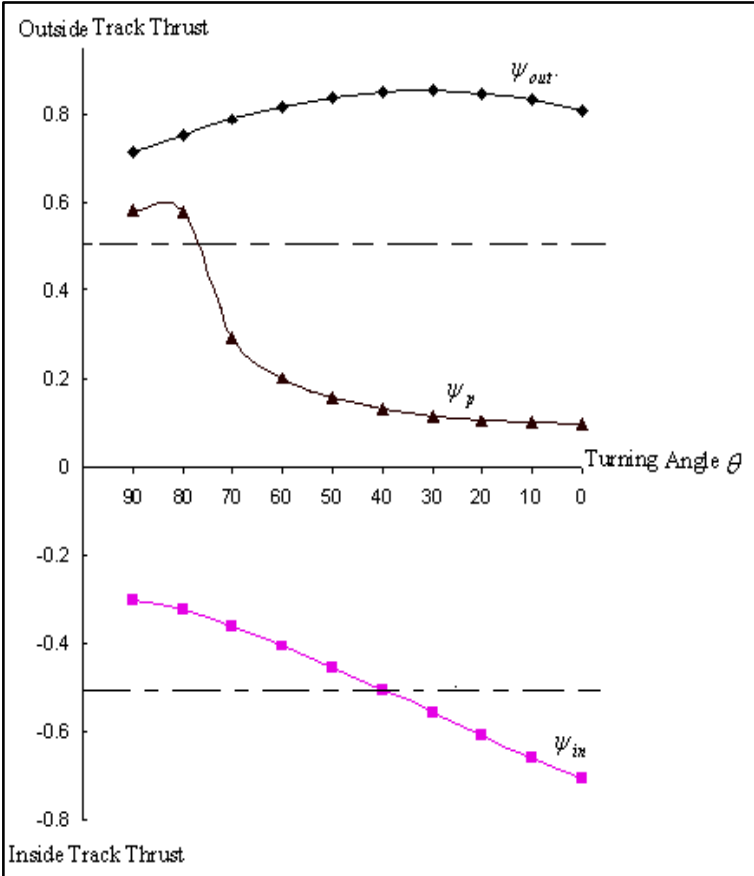

Fig. (12):

Case $6\left(\frac{\mu_{r}}{\mu}=0.1, R_{d l_{1}}=0.5, R_{x h}=1, \frac{W_{1}}{W}=1\right)$ 
Basher: The Influence of Double Acting Hydraulic Piston on Tractive Forces --

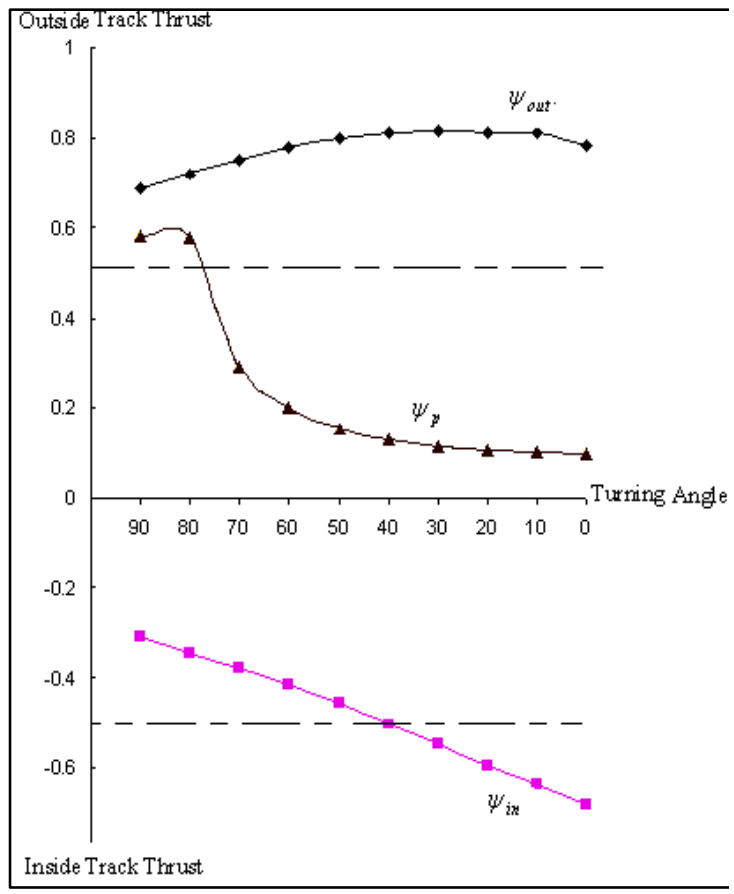

Fig. (13):

Case $7\left(\frac{\mu_{r}}{\mu}=0.1, R_{d l_{1}}=0.6, R_{x h}=1, \frac{W_{1}}{W}=1\right)$

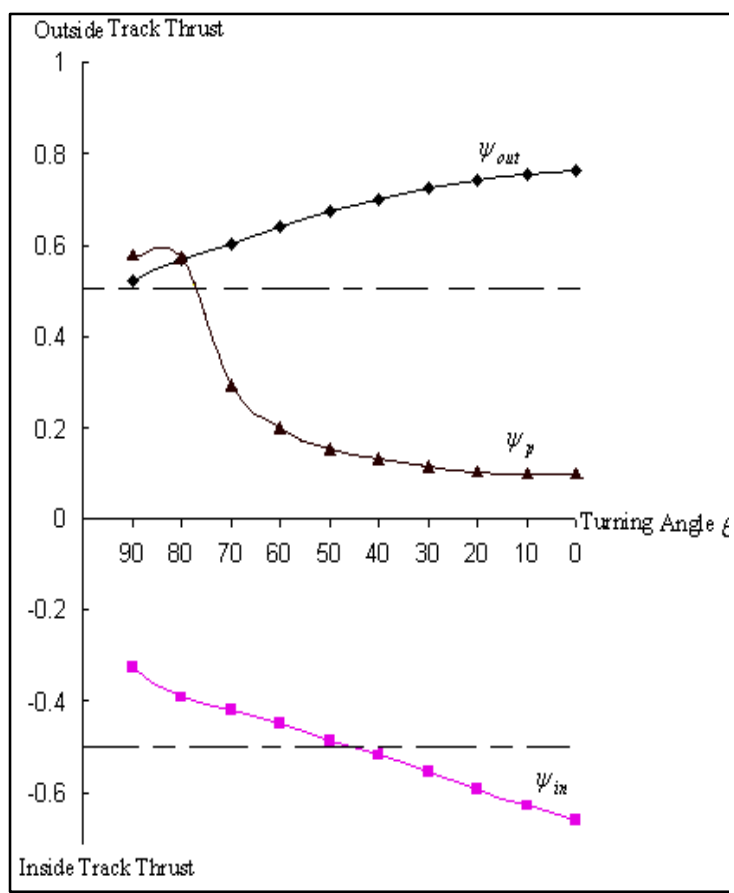

Fig. (15):

Case $9\left(\frac{\mu_{r}}{\mu}=0.1, R_{d l_{1}}=1.5, R_{x h}=1.5, \frac{W_{1}}{W}=1\right)$

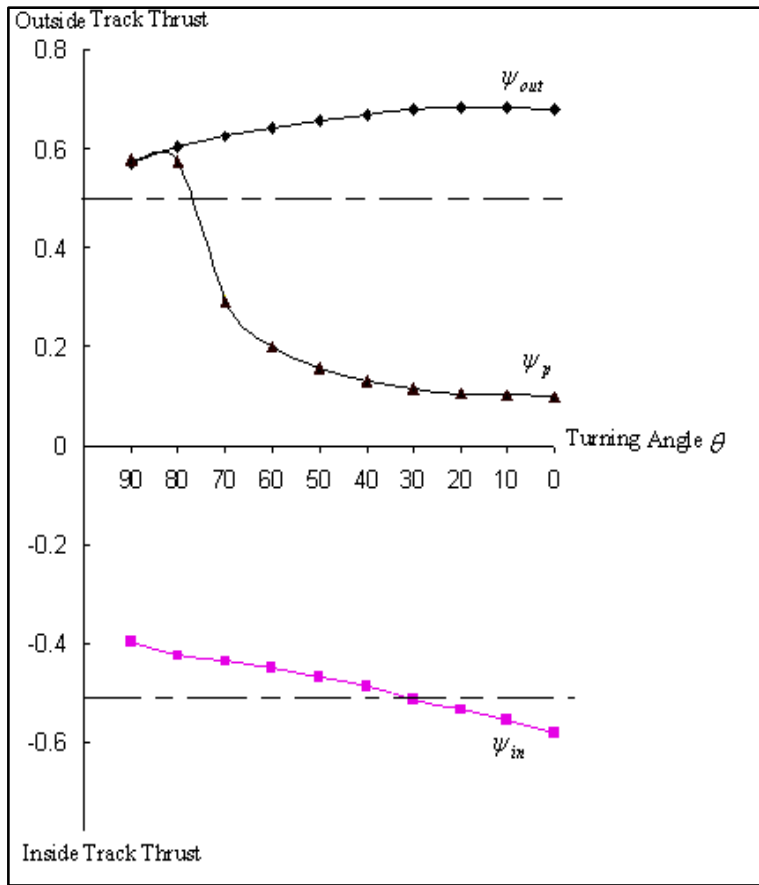

Fig. (14):

Case $8\left(\frac{\mu_{r}}{\mu}=0.1, R_{d l_{1}}=1.5, R_{x h}=1, \frac{W_{1}}{W}=1\right)$

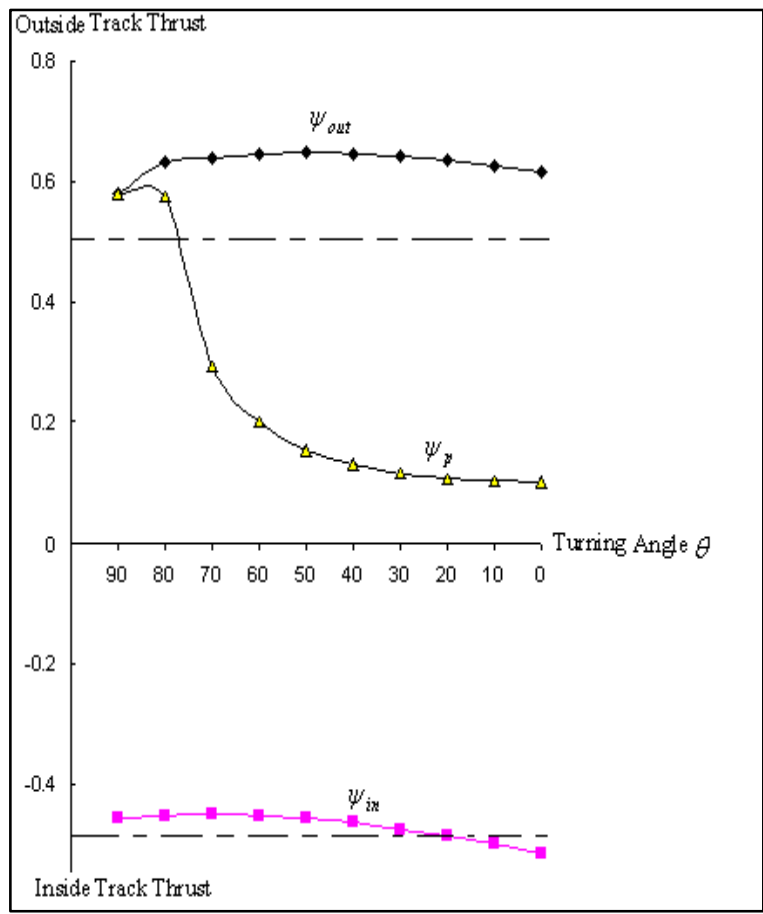

Fig. (16):

Case $10\left(\frac{\mu_{r}}{\mu}=0.1, R_{d l_{1}}=1.5, R_{x h}=0.6, \frac{W_{1}}{W}=1\right)$ 


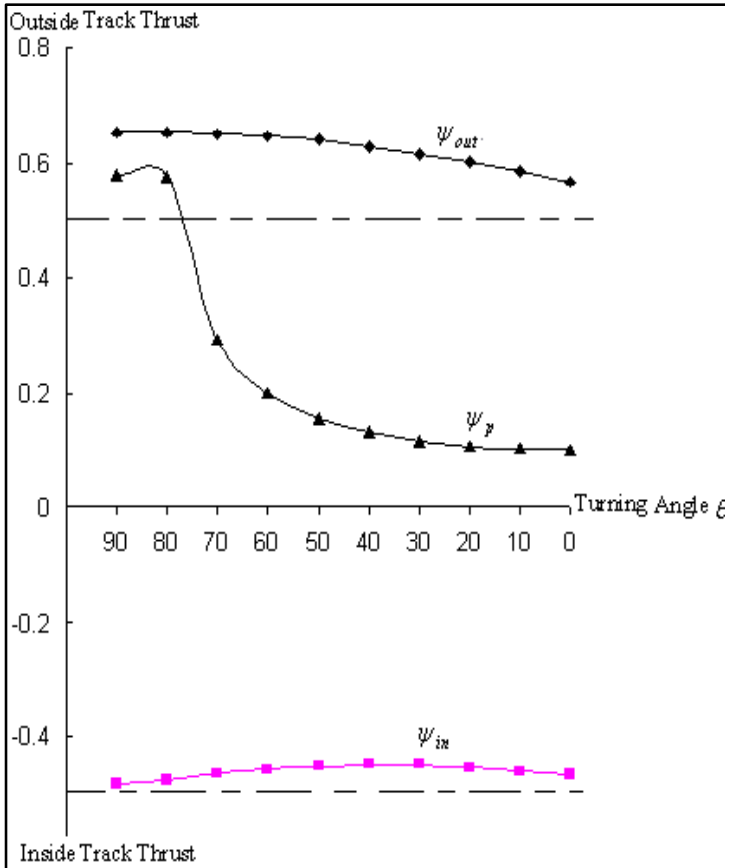

Fig. (17):

Case $11\left(\frac{\mu_{r}}{\mu}=0.1, R_{d l_{1}}=1.5, R_{x h}=0.3, \frac{W_{1}}{W}=1\right)$

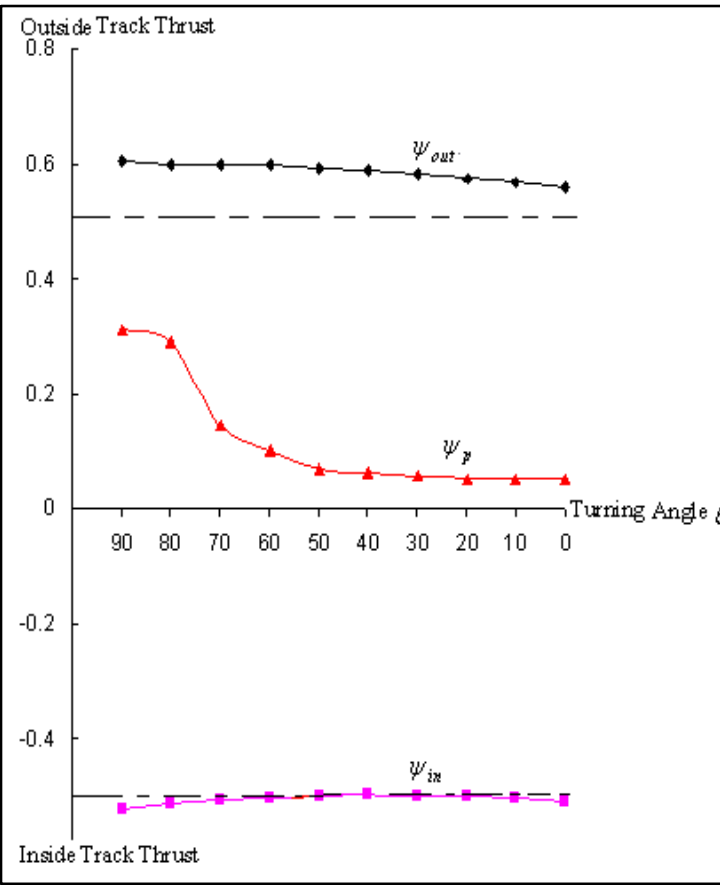

Fig. (19):

Case $13\left(\frac{\mu_{r}}{\mu}=0.1, R_{d l_{1}}=1.5, R_{x h}=0.3, \frac{W_{1}}{W}=0.5\right)$

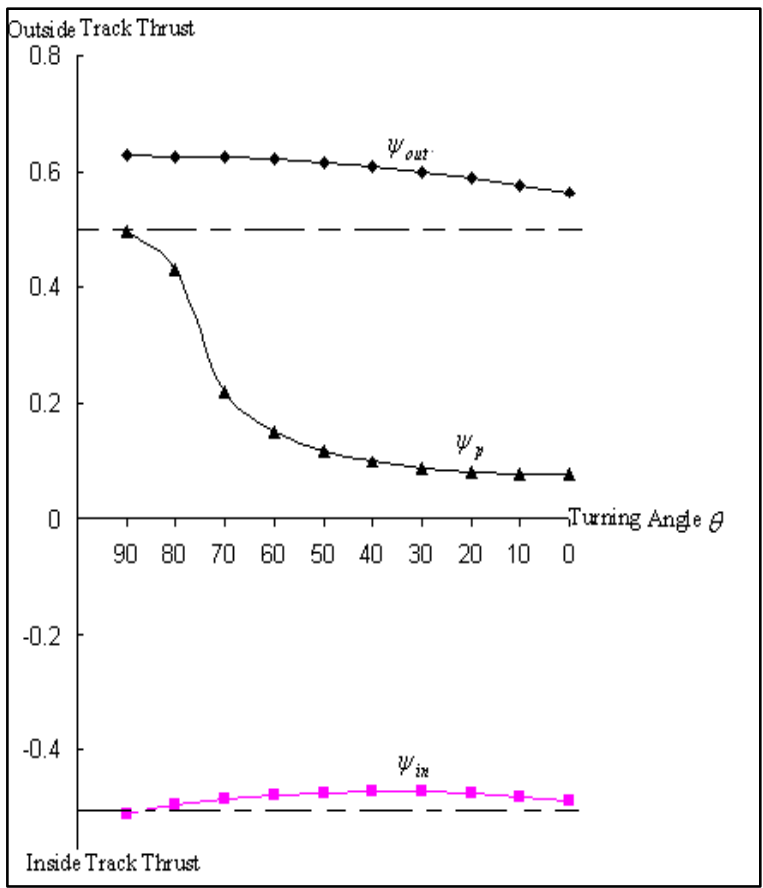

Fig. (18):

$\operatorname{Case12}\left(\frac{\mu_{r}}{\mu}=0.1, R_{d l_{1}}=1.5, R_{x h}=0.3, \frac{W_{1}}{W}=0.75\right)$

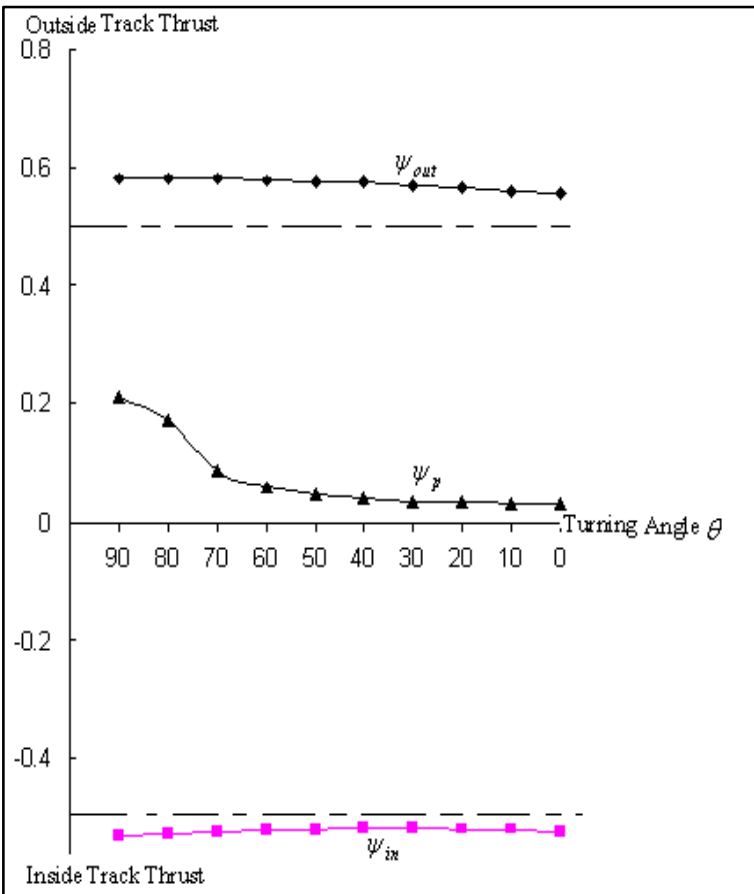

Fig. (20):

Case $14\left(\frac{\mu_{r}}{\mu}=0.1, R_{d l_{1}}=1.5, R_{x h}=0.3, \frac{W_{1}}{W}=0.3\right)$ 


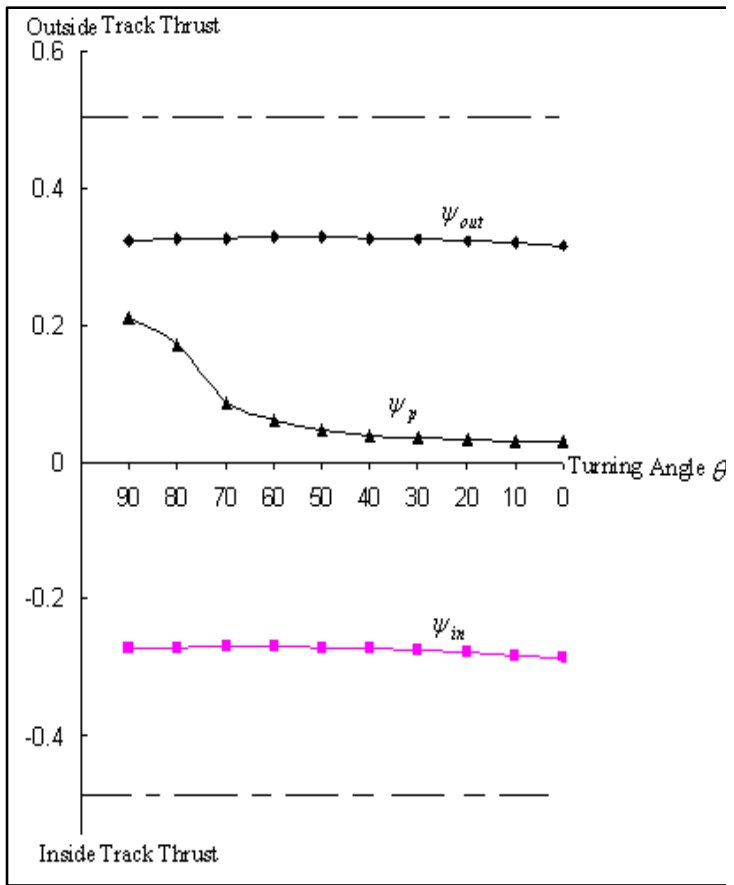

Fig. (21):

Case $15\left(\frac{\mu_{r}}{\mu}=0.1, R_{d l_{1}}=1.5, R_{x h}=0.3, \frac{W_{1}}{W}=0.3, R_{l h}\right.$ $=0.5)$

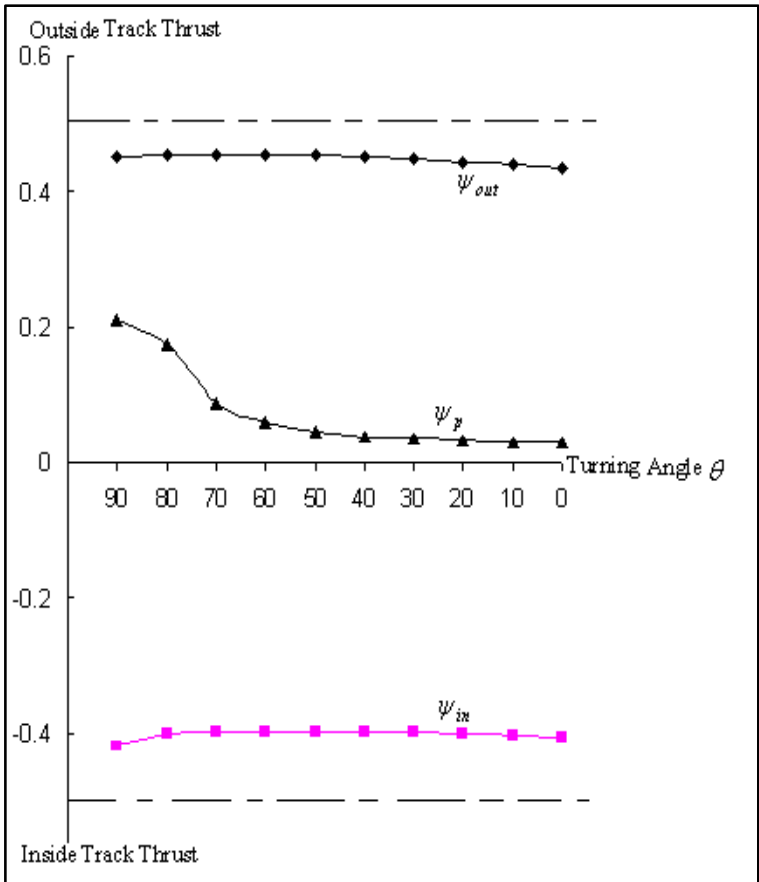

Fig. (22):

Case $16\left(\frac{\mu_{r}}{\mu}=0.1, R_{d l_{1}}=1.5, R_{x h}=0.3, \frac{W_{1}}{W}=0.3, R_{l h}\right.$ $=0.75)$

\section{7- Conclusion:}

Using a double acting hydraulic piston instead of the conventional single acting hydraulic piston is new in the wagon-type articulation of tracked vehicles. It can help in straightening up the position of the two vehicles. This is due to the diminishing effect of hinge force, which is considered as a problem. However, the idea of using a 2-ways hydraulic piston is not recommended when the system is starting to run.

Therefore, the task of the proposed system is to be in action only in straightening up position and not when the system is turning.

The obtained results of this work, i.e the use of double acting hydraulic piston in the wagon -type articulation of tracked vehicles, indicate that the most important factor, which is used in developing the balance of forces is the length to width ratio $R_{l h}$ where smaller value of $l / h,\left(R_{l h} \prec 1\right)$ gives good straightening up. However, there is limitation concerning the dimensions and the shape of the vehicle, which has to be taken in consideration in the design step.

\section{References:}

1- Bekker, M. G., Theory of land locomotion - The Mechanics of Vehicle Mobility, The University of Michigan Press, Ann Arbor, Michigan, 1956.

2- Bekker, M. G., Off-the-Road Locomotion, The University of Michigan Press, Ann Arbor, Michigan, 1960.

3- Bekker, M. G., Introduction to Terrain-Vehicle Systems, The University of Michigan Press, Ann Arbor, Michigan, 1969.

4- Kitano, M. and Jyozaki, H., "A theoretical analysis of steerability of tracked vehicles", J. Terramechanics, Vol. 13, No. 4, pp. 241-258, 1976.

5- Kitano, M. and Kuma, M., "An analysis of horizontal plane motion of tracked vehicles", J. Terramechanics, Vol. 14, No. 4, pp. 211-225, 1977. 
6- Alhimdani,F.F., "Steering analysis of articulated tracked vehicles", J. Terramechanics, Vol. 19, No. 3, pp. 195-209, 1976.

7- Alhimdani,F.F., and Younis,B.A., "Effect of hydraulic piston on hinge force of wagon-type articulated tracked vehicle", J. Terramechanics, Vol. 28, No. 2-3, pp. 137-150, 1991.

8- Alhimdani,F.F.,"Dynamic Analysis of Steering Articulated Tracked Vehicles", Engineering and Development Journal, Vol. 10, No.2, Baghdad, Iraq, June 2006.

9- Ehlert, W., et. al., "Field measurements and analytical models as a basis of test stand simulation of the turning resistance of tracked vehicles", J. Terramechanics, Vol. 29, No. 1, pp 57-69, 1992.

10- Murakami, H., et. al., "A mathematical model for spatial motion of tracked vehicles on soft ground", J. Terramechanics, Vol. 29, No. 1, pp. 71-81, 1992.

11- Shiller, Z. and Serate, W., "Trajectory Planning of Tracked Vehicles", Journal of Dynamic Systems, Measurement, and Control, Vol. 117, pp. 619-624, 1995.

12- Le, A. T., at. al., "Estimation of Track-soil Interactions for Autonomous Tracked Vehicles", Proc. IEEE Int. Conf. on Robotics and Automation, pp. 1388-1393, 1997.

13- Martínez, J.L., et. al, " Kinematic Modelling of Tracked Vehicles by Experimental Identification" International Conference on Intelligent Robots and Systems, Sendai, Japan, pp. 1487-1492, 2004.

14- Martínez, J.L., et. al, " Steering Limitations for a Vehicle Pulling Passive Trailers", IEEE Transactions on Control Systems Technology, Vol.16, No.4, pp. 809-818, 2008.

15- Martínez, J.L., et. al," Virtual Steering Limitations for Reversing an Articulated Vehicle with Off-Axle Passive Trailers", IEEE Transactions on Control Systems Technology, Vol.978, pp. 2385- 2390, 2009.

16-Fijalkowski B.Th.," Novel Mobility and Steerability Enhancing Concept of AllElectricIntelligent Articulated Tracked Vehicles", IEEE Transactions on Control Systems Technology,pp. 225-230, 2003.

17- Govindasamy V., P.Durairaj \& Nivin Joy.T,"Redesign Of Solid Piston Rod of Horizontally Mounted, Free Rod End Type Oil Hydraulic Cylinder Used In Fuel Handling Equipments in Nuclear System Application", IEEE Transactions on Control Systems Technology,pp. 335-338, 2011.

18- Giridhar, "Hydraulics and Pneumatics Hydraulic Cylinder Operating Features-eNotes", Bosch Rexroth (India) Limited-A Drive \& Control Company, 2011. 